This item was submitted to Loughborough's Research Repository by the author.

Items in Figshare are protected by copyright, with all rights reserved, unless otherwise indicated.

\title{
Predicting insulation resistance of enamelled wire using neural network and curve fit methods under thermal aging
}

PLEASE CITE THE PUBLISHED VERSION

https://doi.org/10.1109/ijcnn48605.2020.9207378

PUBLISHER

IEEE

VERSION

AM (Accepted Manuscript)

\section{PUBLISHER STATEMENT}

Personal use of this material is permitted. Permission from IEEE must be obtained for all other uses, in any current or future media, including reprinting/republishing this material for advertising or promotional purposes, creating new collective works, for resale or redistribution to servers or lists, or reuse of any copyrighted component of this work in other works.

\section{LICENCE}

\section{All Rights Reserved}

\section{REPOSITORY RECORD}

Turabee, Gulrukh, Georgina Cosma, Vincenzo Madonna, Paolo Giangrande, Muhammad Raza Khowja, Gaurang Vakil, Chris Gerada, and Michael Galea. 2020. "Predicting Insulation Resistance of Enamelled Wire Using Neural Network and Curve Fit Methods Under Thermal Aging". Loughborough University. https://hdl.handle.net/2134/13061729.v1. 


\section{Predicting Insulation Resistance of Enamelled Wire using Neural Network and Curve Fit Methods Under Thermal Aging}

\author{
Gulrukh Turabee \\ School of Science \& Technology \\ Nottingham Trent University \\ Nottingham, UK \\ Gulrukh.turabee@gmail.com
}

\author{
Muhammad Raza Khowja \\ PEMC Research Group \\ University of Nottingham \\ Nottingham, UK \\ Raza.Khowja@nottingham.ac.uk
}

\author{
Georgina Cosma \\ School of Science \\ Loughborough University \\ Loughborough, UK \\ g.cosma@lboro.ac.uk
}

\author{
Gaurang Vakil \\ PEMC Research Group \\ University of Nottingham \\ Nottingham, UK \\ Gaurang.Vakil@nottingham.ac.uk
}

\author{
Vincenzo Madonna \\ PEMC Research Group \\ University of Nottingham \\ Nottingham, UK \\ ezzvm2@nottingham.ac.uk
}

\author{
Paolo Giangrande \\ PEMC Research Group \\ University of Nottingham \\ Nottingham, UK \\ P.Giangrande@nottingham.ac.uk
}

\author{
Michael Galea \\ Key Laboratory of MEA Technology \\ University of Nottingham \\ Ningbo, China
}

Chris.Gerada@nottingham.ac.uk Michael.Galea@nottingham.ac.uk

\begin{abstract}
Health monitoring has gained a massive interest in power systems engineering, as it has the advantage to reduce operating costs, improve reliability of power supply and provide a better service to customers. This paper presents surrogate methods to predict the electrical insulation lifetime using the neural network approach and three curve fitting models. These can be used for the health monitoring of insulating systems in electrical equipment, such as motors, generators and transformers. The curve fit models and the supervised back propagation neural network are employed to predict the insulation resistance trend of enameled copper wires, when stressed with a temperature of $290{ }^{\circ} \mathrm{C}$. After selecting a suitable end of life criterion, the specimens' mean time-to-failure is estimated, and the performance of each of the analyzed models is apprised through a comparison with the standard method for thermal life evaluation of enameled wires. Amongst all, the best prediction accuracy is achieved by a Backpropagation neural network approach, which gives an error of just $3.29 \%$ when compared with the conventional life evaluation method, whereas, the error is above $10 \%$ for all the three investigated curve fit models.
\end{abstract}

Keywords-Thermal Aging, Neural Network, Curve Fit Models

\section{INTRODUCTION}

Neural networks (NNs) have been applied successfully to solve difficult and diverse problems, including nonlinear system identification and control, financial market analysis, signal modelling, and power load forecasting, by training them in a supervised manner $[1,2]$. It has been proven that NNs can approximate any continuous function as closely as desired. Different system models can be obtained from these networks with proper arrangements of the input variables [3]. A requirement in safety critical applications is the early detection of insulation deterioration, which can be evaluated through a diagnostic property (e.g. insulation capacitance or resistance) of the insulation. In electrical machines, insulation faults have always been a major concern as the majority of stator-windings fail as a result of gradual deterioration of the electrical insulation [4], and the weakest link is often represented by the inter-turn insulation layer, that can trigger the most severe stator faults [5].
Alongside the early detection of abnormal deterioration, another issue is the time required for the experimental test procedure, aiming to determine the lifetime of electrical machines, which can take thousands of testing hours lasting even months [6, 7].

Recent research focuses on the prediction of various nonlinear diagnostic properties (e.g. dielectric loss, tensile strength, elongation, breakdown voltage, insulation resistance and etc), via different NN architectures (e.g. Radial Basis Function Network, Backpropagation, and Long Short-Term Memory). In $[8,9]$, the tensile strength and elongation of XLPE cables were predicted with a Radial Basis Function Network (RBFN) using the Random Optimization Method (ROM) as its training algorithm. Subsequently, the dielectric loss of transformer oil was estimated through a RFBN, however, in this case, other algorithms known as Backpropagation (BP) LevenbergMarquardt (LM) were adopted [10]. The prediction results were in good agreement with the experimental results for all the aforementioned neural network methods, although the BP network produced better results when compared to the LM due to the use of a Bayesian regularization function, which helps the network generalize well and minimise the error [11]. Similarly, Long Short-Term Memory (LSTM), a newly developed recurrent neural network known for its capability of preserving internal memory, was employed by Shpreker et al. [12]. The insulation resistance of electrical equipment was predicted for the early detection of potential failures, whilst also accounting for variations in ambient humidity and air temperature.

According to the existing literature, a NN can be used as an effective tool to provide alternatives to the conventional modelling methodologies, generating attractive results. This is mainly due to the intrinsic characteristics of the technique, such as the ability to automatically learn the relationships between inputs and outputs, independently of the size and complexity of the problem, generalisation capacity and integration facility with other computational tools $[1,2]$. Therefore, this paper proposes the use of BP supervised feed forward neural network, due to its ability to generalise well when predicting the trend of insulation resistance subject to thermal aging. By adopting the neural network approach, it is possible to accurately predict the insulation's diagnostic property, and thus prevent the potential 
insulation failure and subsequent motor outage. Finally, for the purpose of verification, the predicted results have been validated against experimental findings. This paper is structured as follows: section II gives the theoretical background on the diagnostic properties, neural network and curve fit models. In section III, the accelerated thermal aging test procedure, for insulation lifetime evaluation, is discussed. The prediction results, obtained from the neural network and the curve fit methods are presented in section IV, followed by the conclusive remarks in section VI.

\section{THEORETICAL BACKGROUND}

\section{A. Diagnostic Property}

The dissipation factor or dielectric loss (Tan $\delta$ ) is a widely adopted diagnosis test method to evaluate the condition of insulating materials. It provides a good indication of thermal deterioration, moisture absorption and contamination of the test sample, by quantifying the dielectric loss within the insulation [13]. Any insulation system between two adjacent electrical conductors, whose insulation diagnosis is required, can be represented by a capacitance and resistance connected in parallel as shown in Fig. 1. Ideally, any insulating material should behave as a lossless capacitor, indicating the resistance connected in parallel is infinite, i.e. no leakage current flows through the resistor $\mathrm{R}$. This means that, when a sine-wave voltage signal is applied across the capacitor, the resulting current leads by an angle 90 degrees. However, in reality, this is not the case due to the non-idealities of the insulating material. Hence, the leakage current flows through the resistor $\mathrm{R}$, indicating losses within the insulation [14]. The relationship of dissipation factor, in terms of insulation resistance and capacitance, can be obtained by applying the Pythagorean Theorem and Ohm's Law to the phasor diagram in Fig. 1b as in (1) and (2), where $X_{c}$ is the capacitive reactance, and $\omega$ the angular frequency.

$$
\begin{gathered}
\operatorname{Tan} \delta=\frac{I_{R}}{I_{C}} \\
\operatorname{Tan} \delta=\frac{V}{R} \times \frac{X_{c}}{V}=\frac{1}{\omega R C}
\end{gathered}
$$

If the values of dissipation factor and insulation capacitance are known, the insulation resistance, under sinusoidal excitation, can be obtained using (3).

$$
R=\frac{1}{\omega C \operatorname{Tan} \delta}
$$

Generally, the resistive impedance of any insulator is much higher than its capacitive impedance i.e. the $\tan \delta$ is very close to zero. As the aging process goes on, the $\tan \delta$ increases due to the progressive dielectric properties' deterioration leading to an increase in loss, dissipated within the insulation [15]. Fig. 2 shows an example of tan $\delta$ 's trend as effect of thermal aging. The aging mechanism affects this trend in two ways. The first change is an increase in the initial value (for low values of applied voltage), mainly indicating conduction and polarisation losses within the dielectric material. The second change is the rise of dissipation factor at relatively-high voltage. This occurs due to the inception of electrical discharges, in the air gap between two wires, whose magnitude increases as the material ages [16].
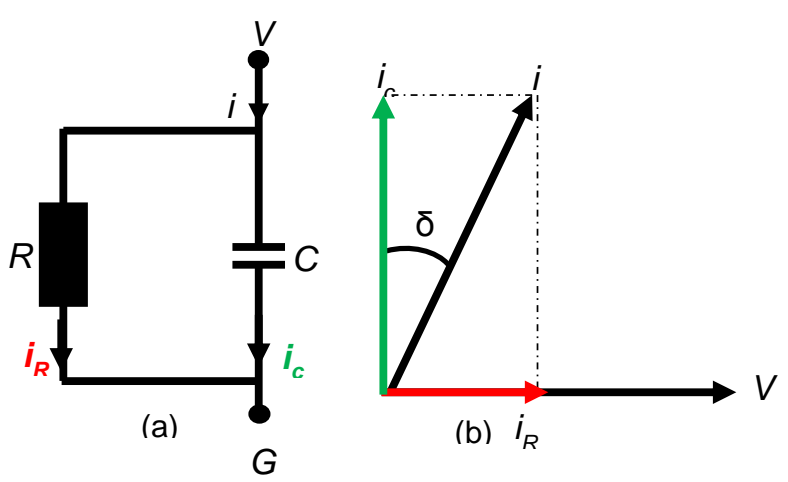

Fig. 1: Non-ideal Dielectric (a) Equivalent circuit (b) Phasor diagram

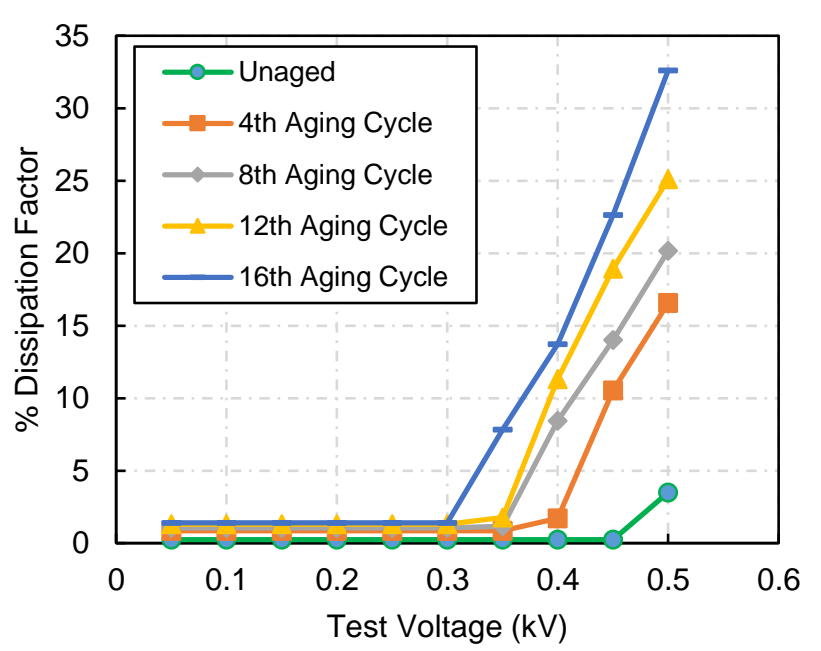

Fig. 2: Dissipation Factor (Specimen \# S9 at $290^{\circ} \mathrm{C}$ ) with respect to the test voltage, from unaged to $16^{\text {th }}$ aging cycle in step of 4

\section{B. BP Neural Network}

BP network is a one-way dissemination and multi-layer forward network, as a result of the use of input-output layer to gradually push the anti-learning algorithm, the error spreads back, referred to as BP network [17]. The network has input and output nodes, with one or multi-nodes implied, but there is no coupling between the nodes in the same layer. The input signal transmits from the input layer nodes in turn through the hidden layer nodes, and reached the final output nodes at the last. Hence, the nodes of each layer can only influence the output of its next layer [18]. The BP network has advantages of simple structure, strong reliability, it is an excellent tool for modeling complex systems. The structure of the BP network is shown in Fig. 3. When a specified training pattern is fed to the input layer, the weighted sum $W$ of the input $X$ to the $j^{\text {th }}$ node in the hidden layer is given by Equation (4) calculating the aggregate input to the neuron. The term $\theta$ is the weighted value from a bias node that always has an output value of 1 .

$$
\mathrm{S}_{m}=\sum_{i=1}^{n} W_{i, m} X_{j m}+\theta
$$




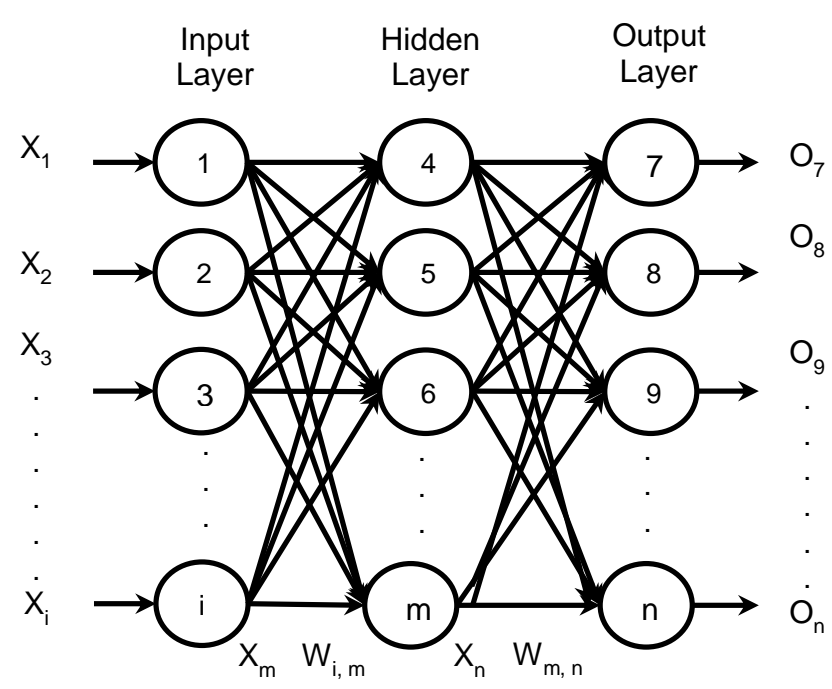

Fig. 3: Architecture of BP Neural Network with one hidden layer

Action potential of neuron is decided by an appropriate activation function (i.e. sigmoid function as shown in Equation (5)). The resulting value from the activation function determines the neuron's output and becomes the input value for the neurons in the next layer connected to it.

$$
O_{m}=X_{n}=\frac{1}{1+e^{-S_{m}}}
$$

Considering the actual activation value of the output node, $n$, is $O_{n}$ and the target value is $t_{n}$, then the difference between the actual output and expected output is given by (6).

$$
\Delta_{n}=t_{n}-O_{n}
$$

Equation (7) is used to modify the weight, $W_{m, n}$, between the output node, $n$, and the node, $m$, where $\Delta W_{m, n}$ is the change in the weight between nodes $\mathrm{m}$ and $\mathrm{n}$ and $l_{r}$ is the learning rate.

$$
\Delta W_{m, n}=l_{r} X_{n} \Omega_{n}
$$

$\Omega_{n}$ is the error signal for node in the output layer and can be represented by (8).

$$
\Omega_{n}=\Delta_{n} O_{n}\left(1-O_{n}\right)
$$

\section{Curve Fit Models}

Curve fit models, known as regression analysis models, are commonly used to find the "best fit" for a series of data points, in the form of an equation that can be adopted to estimate the points anywhere along the curve. Therefore, by exploiting curve fit models, the diagnostic properties of the insulation are predicted along with its time-to-failure in next sections. The time-to-failure prediction is a pre-requisite for insulation lifetime prediction modelling purposes. Three different curve fit models are considered in this paper, in order to predict the insulation's lifetime at $290^{\circ} \mathrm{C}$ aging temperature, the details of which are enlisted below:

- Exponential function fits a curve through a given set of data (e.g. any diagnostic property of insulating material), as described by (9). It is often adopted when the rate of change of a quantity is proportional to the initial amount of the quantity. If the coefficient " $\mathrm{B}$ " is negative, the function exponentially decays whereas, if " $b$ " is positive, then y represents exponential growth.

- Logarithmic function fits a curve through a given dataset in the form of (10). For this case, if the coefficient " $\mathrm{m}$ " is negative, the function represents logarithmic decay whereas, if the coefficient " $\mathrm{m}$ " is positive, the function represents a logarithmic rise.

- Power series function fits a curve through a given dataset in the form of (11). In this case, if the coefficient " $b$ " is negative, the function represents a decay through a decreasing power law whereas, if " $b$ " is positive, the function rises through a positive power law.

The aforementioned curve fit models are suited for fitting positive data.

$$
\begin{gathered}
y(t)=A e^{B t} \\
y(t)=m \cdot \ln (t)+c \\
y(t)=a t^{b}
\end{gathered}
$$

\section{THERMAL AgING TESTS}

\section{A. Test Object}

The specimen holder is fitted with 10 unaged twisted pair, made of round enamelled copper wires, without any varnish or resin applied over them, as shown in Fig. 4a. The wire features a the double layer of enamel i.e. a modified-polyester base coat which is top coated with a polyamide-imide material, which enhances the wire's thermo-mechanical properties (i.e. better resistance to heat and abrasion as opposed to single coated wires). The wire has a (declared) thermal class of $220^{\circ} \mathrm{C}$ (i.e. temperatures which guarantees 20,000 operating hours). The specimens are prepared according to the technical standard ASTM D2307 [19]. Such wire type is mostly adopted for the winding voltage less than $1 \mathrm{kV}$, in electro-magnetic components such as electic motors, transformers and inductors [20-23] .

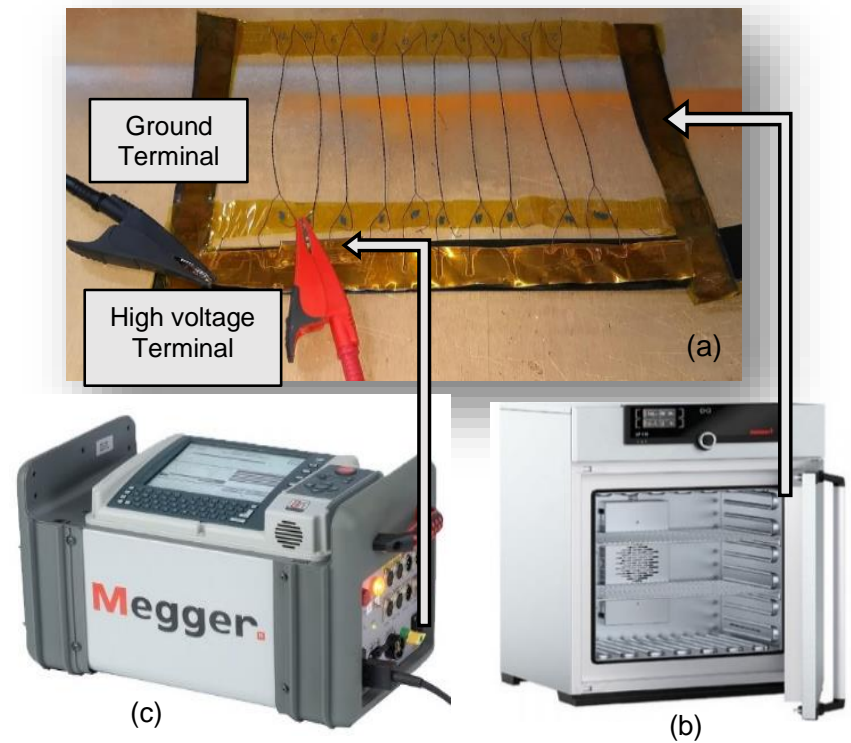

Fig. 4: Measurement Setup (a) Specimen's Holder in an unaged condition (b) MEMMERT UF260 Oven (c) Diagnostic Device MEGGER 4000 


\section{B. Test Setup and Procedure}

During the thermal aging campaign, the specimens are stressed through constant temperature above their insulation thermal class. For a given twisted pair, the aging procedure ends when the end of life is reached, i.e., when the dielectric breakdown is detected. The thermal aging tests were conducted under the thermal stress of $290^{\circ} \mathrm{C}$, which is $70^{\circ} \mathrm{C}$ above the thermal class. The specimen holder was heated-up in a controlled oven (Fig. 4b) that regulates the inside temperature up to $300^{\circ} \mathrm{C}$. The diagnostic properties, such as dissipation factor and insulation capacitance, were measured, whereas the insulation resistance of every twisted-pair wire was calculated using (3), and it is used for the prediction analysis in the forthcoming sections. The diagnostic properties of the turn-toturn insulation were measured through a MEGGER ${ }^{\circledR}$ Delta 4000 (Fig. 4c), after every 8 hours of $290^{\circ} \mathrm{C}$ thermal exposure. During the diagnosis session, the test voltage is applied across the insulation of each analysed specimens (i.e. across the terminals of each twisted pair). Once the insulation breakdown is detected in a specimen, its time-to-failure is noted. The test campaign then ends when all the specimens have failed. After competition of the test procedure, the failure times are statistically post processed.

\section{Prediction Using BP NeURAL NeTwork}

\section{A. Training Stage}

The training phase involves data learning, by presenting it to the input layer and adjusting the various parameters of the NN to achieve the desired output value. As mentioned earlier, BP was used to train the given dataset provided $\left(X_{i}, X_{i+1}\right)$ where $X_{i}$ represents the value of diagnostic parameter at aging time $t_{i}$ and $\mathrm{X}_{\mathrm{i}+1}$ represents its prediction at aging time $\mathrm{t}_{\mathrm{i}+1}$. The insulation resistance, along with the aging time was presented to the $\mathrm{NN}$ as input.

\section{B. Prediction Stage}

The prediction phase involves testing of NN on unseen data, where this data is presented to the network as an input which is processed by the network to produce an output, thereby predicting the output of unseen input data. In order to obtain the best prediction outcome, selecting optimal parameters (i.e. number of neurons) and number of data points for training and prediction phase often plays a pivotal role [8]. Thus, different number of data points and number of neurons were experimented at the training stage. It was noticed that the best results were produced when the given dataset was trained with 8 learning points (i.e. until $56 \mathrm{~h}$ aging time) in combination with 5 number of neurons. As the latest data either predicted or trained is crucial for the model to learn and produce the optimal upcoming outcome. Therefore, a unique training strategy was applied in which once the training has been performed initially (on 8 data points), then, the next value ( $9^{\text {th }}$ data point) has been predicted. The latter predicted value was included in the training dataset for the prediction of the forthcoming value $\left(10^{\text {th }}\right.$ data point). In this way, each time a value was predicted, it was included in the training dataset for the prediction of upcoming value. This strategy helped the NN to learn the features of latest data point predicted. Fig. 5 shows a flowchart summarising the applied strategy.

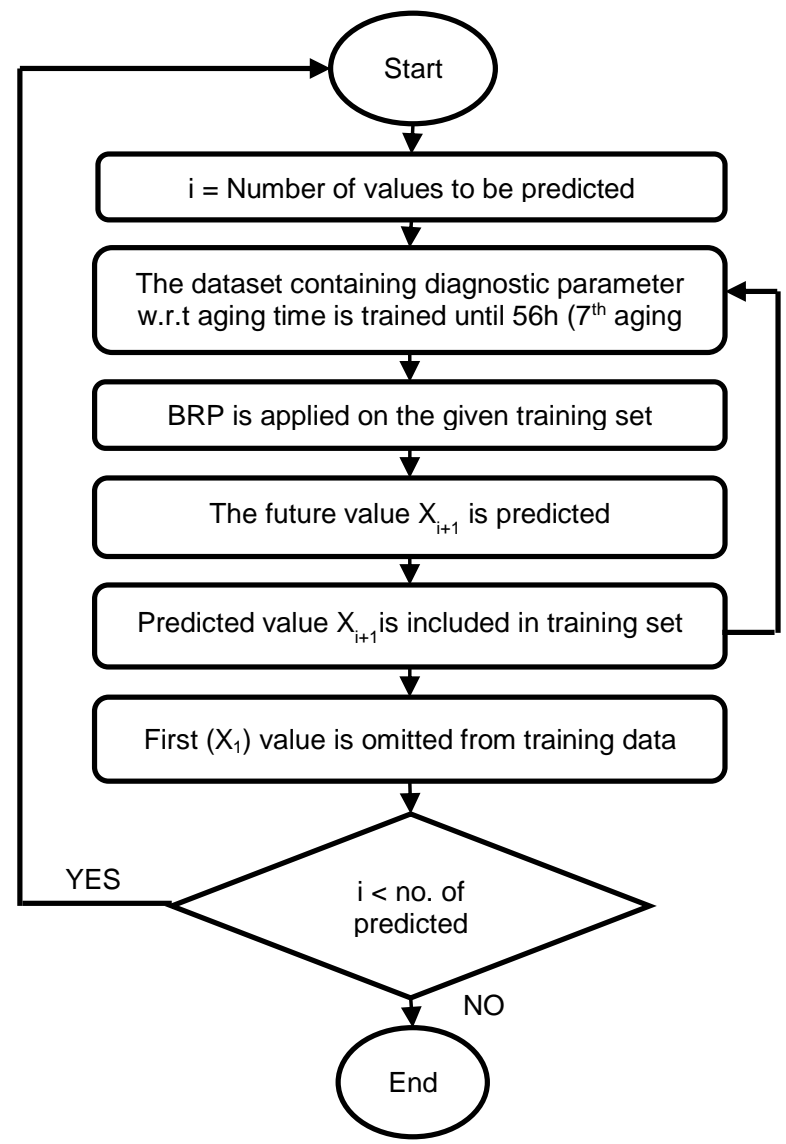

Fig. 5: Flowchart of Training and Prediction Strategy

\section{RESULTS AND DISCUSSIONS}

Fig. 6(a) to (d) illustrate the prediction results of 4 specimens (S1, S3, S4 and S8 respectively) out of 10, acquired through BP network and three CF methods (i.e. Exponential, Logarithmic and Power Series) respectively. For the purpose of a fair comparison, the CF models use the data points (insulation resistance vs aging time) until $56 \mathrm{~h}$ to generate the fitting equation. The insulation resistance data is then extrapolated until $160 \mathrm{~h}$, using the same equation. The predicted results of all 4 methods are then compared with the experimental data collected during the test procedure according to the discussion of section III. The regression plot, error analysis, time-to-failure and mean time-to-failure of the specimens under thermal aging are presented in the subsections below.

\section{A. Regression Plot}

For the purpose of validating the results obtained through the adopted approaches, the regression plots of the BP network and the exponential CF are computed and they are shown in Fig. 7 and Fig. 8 respectively. These plots represent the relationship between outputs and targets for the selected approaches. It can be noticed that the BP network results present a good fit with an $\mathrm{R}$ value of 0.99927 ( $\approx$ unity), thus indicating a linear relationship between the targets and the outputs. Whilst, for the exponential CF regression plot (Fig.8), a slightly scattered plot indicates that certain data points have a poor fit, with an $\mathrm{R}$ value of 0.9956. Here, the dotted line in each plot represents the desired values and the solid line highlights the best fit linear regression line between outputs and the targets. 

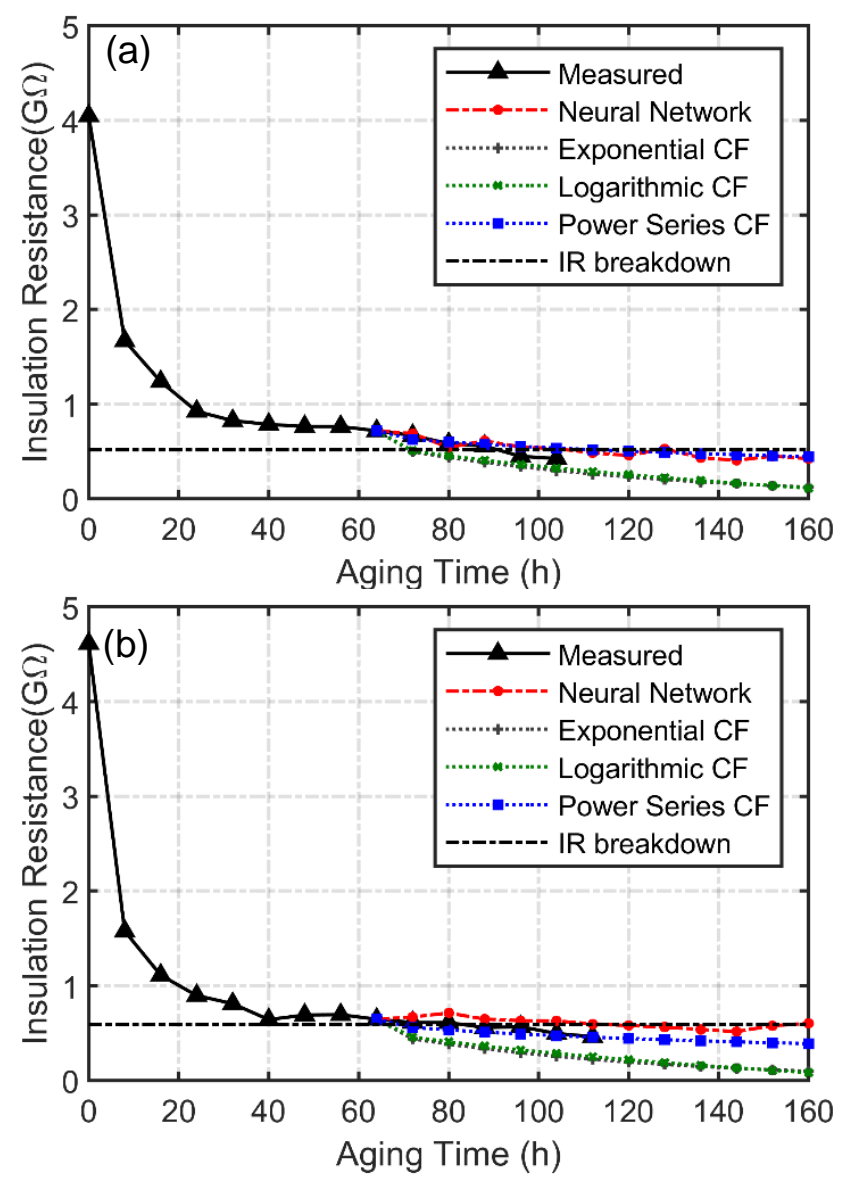

Fig. 6: IR Prediction Results (a) S1 (b) S3 (c) S4 (d) S8

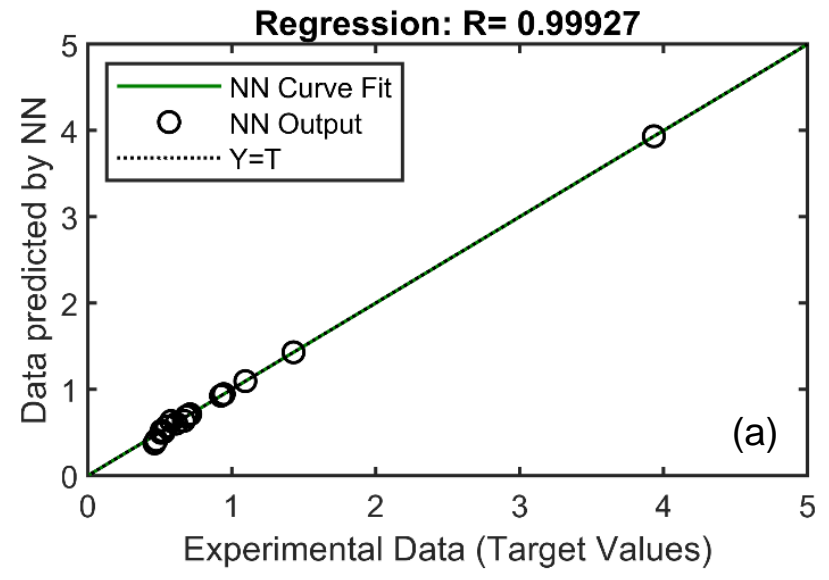

Fig. 7: Regression Plots (a) BP Network and (b) Exponential CF

\section{B. Relative Error of proposed approaches}

For the purpose of illustration and comparison of predicted insulation resistance, the percentage error was calculated for all of the 4 analysed methodologies as shown in Fig. 8. Specimen S9 has been chosen for this task since it survived for a longer time (i.e. failed at 136h). It can be seen that the BP network presents the lowest error of approximately 5\% (on average). On the contrary, the exponential CF approach shows the highest relative error of $45 \%$. For the remaining two methods, the percent error is in-between the NN and the exponential CF.
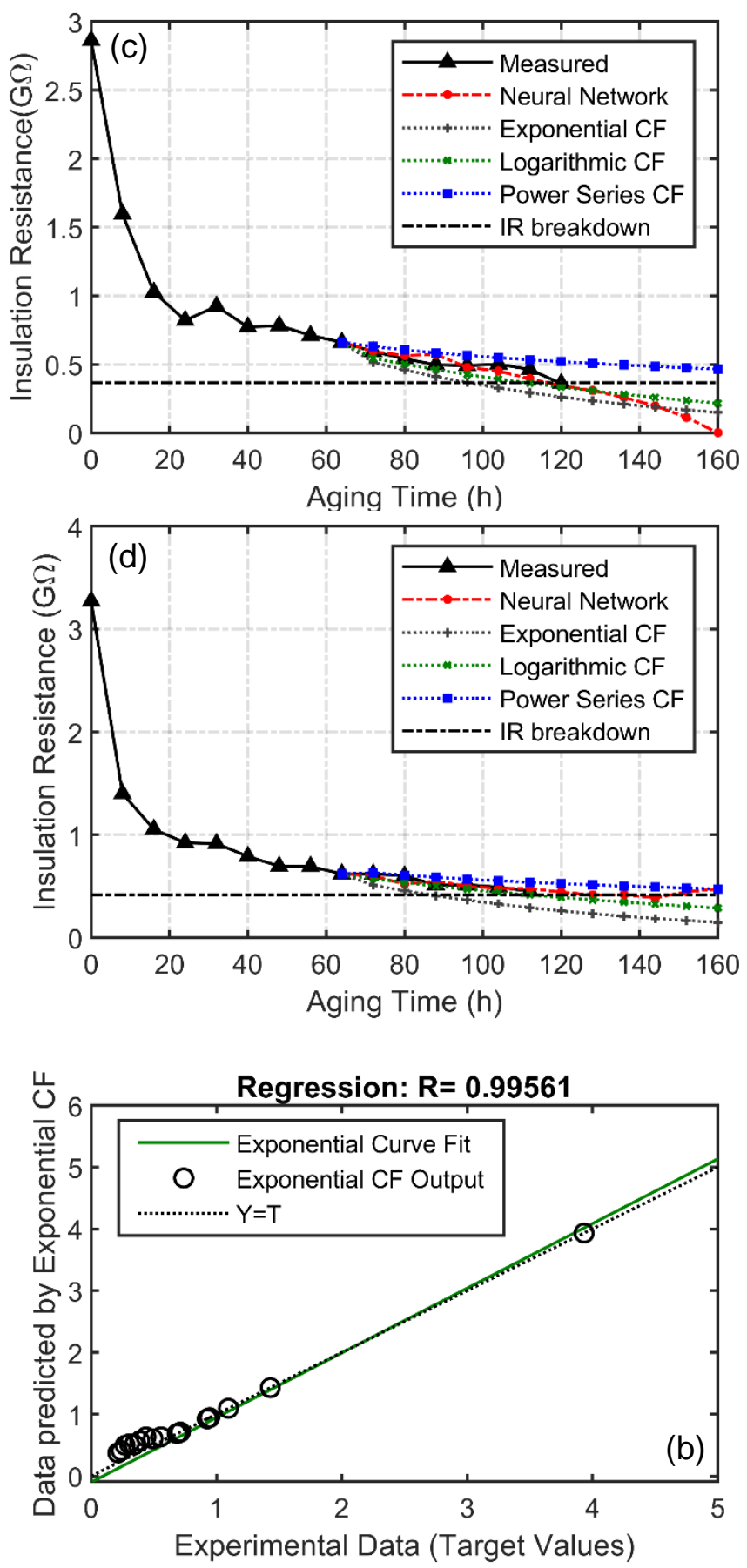

C. Regression Error Metrics

Table I tabulates three different regression errors and the Rsquare (Coefficient of Determination) for further apprising the prediction quality of the three presented methods. Here, the Mean Absoluter Error (MAE) indicates the absolute difference between the experimented data and all 4 used model's predictions. It can be observed that the power CF followed by $\mathrm{BP}$ network is characterised by the lowest MAE, which is less than 0.05 , whilst the exponential CF has an error greater than 0.1 . Here, a small MAE suggests model's ability to predict well. 


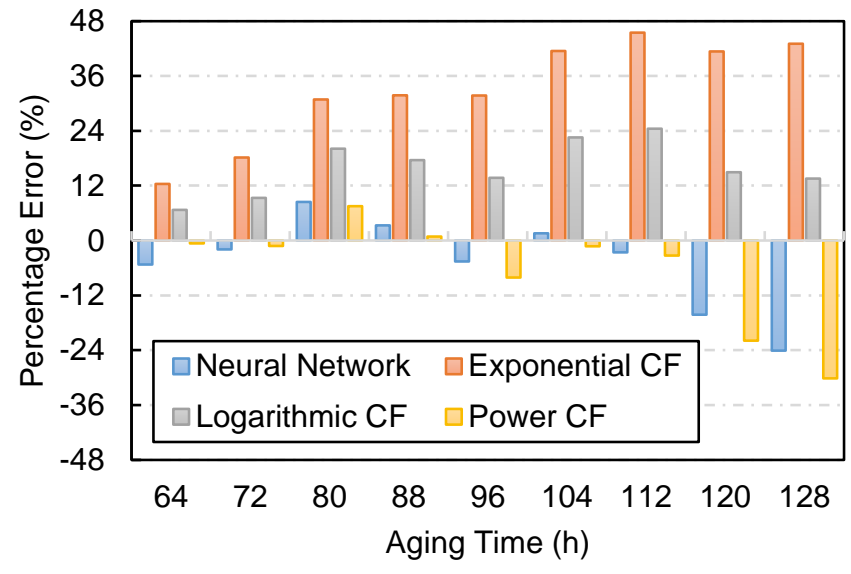

Fig. 8: Percentage Error Comparison of Specimen S9

TABLE I. REGRESSION ERRORS AND R-SQ

\begin{tabular}{|c|c|c|c|c|}
\hline $\begin{array}{l}\text { Error } \\
\text { Category }\end{array}$ & $\begin{array}{l}\text { BP } \\
\text { NN }\end{array}$ & $\begin{array}{c}\text { Exponential } \\
\text { CF }\end{array}$ & $\begin{array}{c}\text { Logarithmic } \\
\text { CF }\end{array}$ & $\begin{array}{c}\text { Power } \\
\text { CF }\end{array}$ \\
\hline RMSE & 0.044 & 0.172 & 0.089 & 0.053 \\
\hline MAPE & 7.556 & 32.92 & 15.89 & 8.322 \\
\hline MAE & 0.035 & 0.166 & 0.083 & 0.037 \\
\hline $\mathrm{R}^{2}$ & 0.9985 & 0.9912 & 0.9973 & 0.9981 \\
\hline
\end{tabular}

RMSE: Root Mean Square Error, MAPE: Mean Absolute Percentage Error, MAE: Mean Absolute Error

However, it fails to identify the underperformance or overperformance of the used model. Thus, the Root Mean Square Error (RMSE), indicating how large the residuals are spread out has been calculated. It shows an error of about 0.044 and 0.172 for the $\mathrm{BP}$ network and the exponential $\mathrm{CF}$ respectively. Next, the Mean Absolute Percentage Error (MAPE) was estimated, demonstrating how far the model's predictions are from their corresponding target values on average. Approximately, $7 \%$ was the minimum error determined for all aging cycles for the BP approach followed by a $16 \%$ error for the logarithmic CF method and $33 \%$ being the maximum error for the exponential CF method. Finally, the goodness of fit, also known as coefficient of determination $\left(\mathrm{R}^{2}\right)$, was estimated. It is a method to find the better fit. In other words, it is a measure of "how well" a model can predict the data, and falls between 0 and 1 . The higher the value of $R^{2}$, the better the model prediction is. For BP network the value of $\mathrm{R}^{2}$ is 0.9985. Being the highest amongst the presented methods, clearly shows that the BP network fits the data well. It is followed by the power CF approach with a value of 0.9981 . For the logarithmic and the exponential CF, the $\mathrm{R}^{2}$ is 0.9973 and 0.9972 respectively, indicating that both methods do not provide a good fit.

\section{Specimen's Time-to-Failure}

In order to forecast the time-to-failure of each thermally aged specimen, an end of life breakdown criterion is chosen. The latter is fixed as an $87.77 \%$ variation in the monitored diagnostic marker [6]. This implies that the specimen's life comes to an end when its insulation resistance, under thermal aging, is reduced by $87.77 \%$ from its unaged value. This can be seen in Fig. 6(a)(d) where the black dotted line represents the
TABLE II. SPECIMEN'S TIME-TO-FAILURE COMPARISON

\begin{tabular}{lccccc}
\hline Specimen \# & CM & NN & LCF & ECF & PCF \\
\hline S1 & 108 & 110 & 74 & 73 & 116 \\
S2 & 100 & 82 & 86 & 73 & 117 \\
S3 & 116 & 117.3 & 47.6 & 58.6 & 68.1 \\
S4 & 124 & 118 & 115 & 100 & 176 \\
S5 & 116 & 86 & 86 & 68 & 118 \\
S6 & 84 & 127.1 & 96.3 & 81 & 147.2 \\
S7 & 100 & 123 & 157 & 106 & 180 \\
S8 & 116 & 119 & 110 & 90 & 180 \\
S9 & 132 & 116 & 83 & 75 & 118 \\
S10 & 68 & 94 & 66 & 61 & 77 \\
\hline CM: Conventional Method, NN: BP Neural Network, LCF: Logarithmic \\
CF, ECF: Exponential CF, PCF: Power CF
\end{tabular}

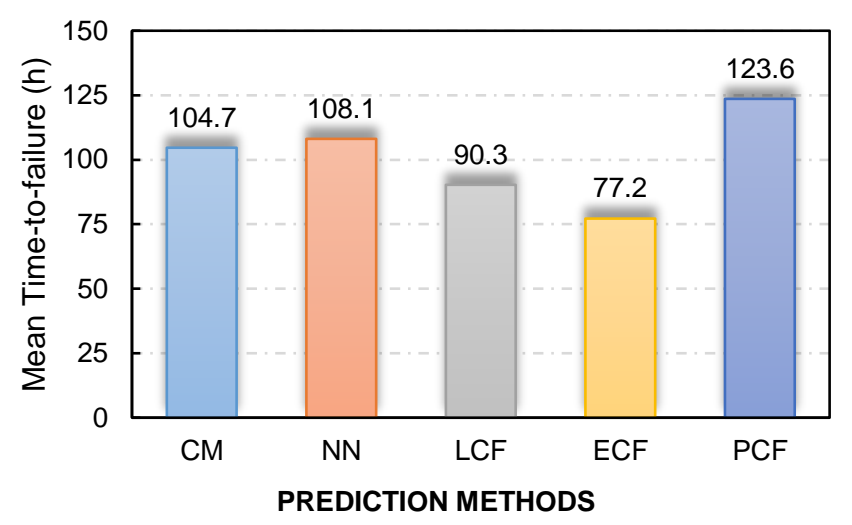

Fig. 9 Comparison of Mean Time-to-failure

end of life threshold value of the insulation resistance ( $\left.\mathrm{IR}_{\text {breakdown }}\right)$. In this way, the time-to-failure of each specimen is noted down, based on the chosen breakdown criterion as listed in Table II. For the conventional method, the time-to-failure is evaluated as - the total time of thermal exposure minus half duration of one aging cycle (i.e. the insulation breakdown is assumed to occur at half of the last thermal cycle). However, for the considered methods (neural network and curve fit models), the time-to-failure is calculated as - the time at which the breakdown criteria is reached. The mean time-to-failure, as shown in Fig. 9, is calculated using log average method as described in the American Standard ASTM D2307 [19]. The standard suggests to calculate the time to failure by dividing the sum of the logarithms of the failure times of the individual specimens at each aging temperature by the total number of specimens in the group. The failure time of the group is the antilogarithm of the logarithmic mean. This is represented by (12).

$$
M T T F=\operatorname{Antilog}\left(\frac{1}{N} \sum_{i=1}^{N} \log _{10}(T T F)\right)
$$

In terms of insulation resistance prediction accuracy, both $\mathrm{NN}$ and logarithmic $\mathrm{CF}$ give promising results, as compared to exponential $\mathrm{CF}$ and power $\mathrm{CF}$. Both $\mathrm{NN}$ and logarithmic $\mathrm{CF}$ follow the measured insulation resistance very closely. However, premature breakdown is noticed in the exponential CF whereas, the specimens have failed comparatively late, when predicted using the pPower CF (Fig. 6c and Fig 6d). Due to the delayed breakdown predicted using the power $\mathrm{CF}, 2$ specimens have not reached the breakdown threshold until 160h i.e. S7 and S8 (see underlined specimens in Table II). Therefore, these 2 
specimens were deliberately censored at $180 \mathrm{~h}$, in order to determine the power CF's mean time-to-failure. In terms of mean time-to-failure accuracy, the NN provides the best match, when compared to the conventional "standard" method, giving a percentage error of just $3.29 \%$ as opposed to the logarithmic $\mathrm{CF}$, exponential $\mathrm{CF}$ and power $\mathrm{CF}$, whose errors are $13.7 \%$, $26.3 \%$ and $18.1 \%$ respectively.

\section{CONCLUSIONS}

Surrogate approaches to estimate the lifetime of enamelled wire insulation, under thermal aging, are presented in this paper. The insulation's life has been predicted using a supervised neural network and three curve fit models i.e. logarithmic $\mathrm{CF}$, exponential $\mathrm{CF}$ and power $\mathrm{CF}$. The insulation resistance of each specimen was predicted under thermal exposure of $290^{\circ} \mathrm{C}$. For specimen S9, which lasted the longest in the test campaign, the regression error analysis was carried out in terms of RMSE, MAPE, MAE and $\mathrm{R}^{2}$. Amongst all prediction methods, the neural network gives the best results in terms of prediction accuracy. Regarding the mean time-to-failure prediction accuracy, the neural network method has made the best match, when compared to the conventional method provided by technical standards, as it gives a percentage error of $3.29 \%$. On the contrary the curve fit methods, i.e. logarithmic $\mathrm{CF}$, exponential $\mathrm{CF}$ and power $\mathrm{CF}$ feature errors of $13.7 \%, 26.3 \%$ and $18.1 \%$ respectively. Since only 7 to 8 data points (until $56 \mathrm{~h}$ aging time) have been used in the neural network and curve fit methods, a large amount testing time could potentially be saved when the insulation's lifetime is evaluated at aging temperatures lower than $290^{\circ} \mathrm{C}$ (such as $270^{\circ} \mathrm{C}$ and $250^{\circ} \mathrm{C}$ ). Thus, the proposed approaches show clear benefits in terms of time-saving during the experimental test procedure (i.e. conventional method), where at least three aging temperatures are required to complete the process of thermal qualification [6, 19]. Additional results, regarding different aging temperatures will be addressed in separate publications.

\section{REFERENCES}

[1] G. Turabee, Y. Shen and G. Cosma, "Interpreting the Filters in the First Layer of a Convolutional Neural Network for Sleep Stage Classification," in UK Workshop on Computational Intelligence, pp. 142-154, 2019.

[2] S. Haykin and N. Network, "A comprehensive foundation," Neural Networks, vol. 2, pp. 41, 2004

[3] T.K. Gupta and K. Raza, "Optimization of ANN Architecture: A Review on Nature-Inspired Techniques," in Machine Learning in Bio-Signal Analysis and Diagnostic Imaging, Elsevier, 2019, pp. 159-182.

[4] Y. Han and Y.H. Song, "Condition monitoring techniques for electrical equipment-a literature survey," IEEE Trans.Power Del., vol. 18, pp. 4-13, 2003.

[5] V. Madonna, P. Giangrande, L. Lusuardi, A. Cavallini, C. Gerada and M. Galea, "Thermal overload and insulation aging of short duty cycle, aerospace motors," IEEE Trans.Ind.Electron., 2019.

[6] G. Turabee, M. Khowja, P. Giangrade, V. Madonna, G. Cosma, G. Vakil, C. Gerada and M. Galea, "The Role of Neural Networks in Predicting the Life of Electrical Machines," IEEE Access, 8, pp.40283-40297.

[7] V. Madonna, P. Giangrande, G. Migliazza, G. Buticchi and M. Galea, "A Time-Saving Approach for the Thermal Lifetime Evaluation of Low Voltage Electrical Machines," IEEE Trans.Ind.Electron., 2019.
[8] L. Boukezzi and A. Boubakeur, "Prediction of mechanical properties of XLPE cable insulation under thermal aging: neural network approach," IEEE Trans.Dielectr.Electr.Insul., vol. 20, pp. 2125-2134, 2013.

[9] L. Bessissa, L. Boukezzi, D. Mahi and A. Boubakeur, "Lifetime estimation and diagnosis of XLPE used in HV insulation cables under thermal ageing: arithmetic sequences optimised by genetic algorithms approach," IET Generation, Transmission \& Distribution, vol. 11, pp. 2429-2437, 2016.

[10] L. Mokhnache, A. Boubakeur and N.N. Said, "Comparison of different neural networks algorithms used in the diagnosis and thermal ageing prediction of transformer oil," in IEEE International Conference on Systems, Man and Cybernetics, pp. 6 pp. vol. 6, 2002.

[11] Bate, A., Lindquist, M., Edwards, I.R., Olsson, S., Orre, R., Lansner, A. and De Freitas, R.M., 1998. A Bayesian neural network method for adverse drug reaction signal generation. European journal of clinical pharmacology, 54(4), pp.315-321 .

[12] D.M. Shprekher, G.I. Babokin and E.B. Kolesnikov, "Application of Neural Networks for Prediction of Insulation Condition in Networks with Isolated Neutral," in 2019 International Russian Automation Conference (RusAutoCon), pp. 1-6, 2019.

[13] N. Phloymuk, P. Nimsanong, N. Phumipunepon, S. Potivejkul, T. Wiangtong and N. Pattanadech, "The Dissipation Factor ( $\tan \delta$ ) Monitoring of A Stator Winding Insulation of A Synchronous Machine," in 2018 Condition Monitoring and Diagnosis (CMD), pp. 1-4, 2018.

[14] V. Madonna, P. Giangrande and M. Galea, "Evaluation of strand-to-strand capacitance and dissipation factor in thermally aged enamelled coils for lowvoltage electrical machines," IET Science, Measurement \& Technology, vol. 13, pp. 1170-1177, 2019.

[15] K.N. Gyftakis, M. Sumislawska, D.F. Kavanagh, D.A. Howey and M.D. McCulloch, "Dielectric characteristics of electric vehicle traction motor winding insulation under thermal aging," IEEE Trans.Ind.Appl., vol. 52, pp. 1398-1404, 2015.

[16] M. Farahani, E. Gockenbach, H. Borsi, K. Schafer and M. Kaufhold, "Behavior of machine insulation systems subjected to accelerated thermal aging test," IEEE Trans.Dielectr.Electr.Insul., vol. 17, pp. 1364-1372, 2010.

[17] J. Zhao, H. Jin and H. Han, "Dielectric Loss Factor Forecasting Based on Artificial Neural Network," in 2009 Second International Conference on Information and Computing Science, pp. 177-180, 2009.

[18] R. Hecht-Nielsen, "Theory of the backpropagation neural network," in Neural networks for perception, Elsevier, 1992, pp. 65-93.

[19] Anonymous "Standard Test Method for Thermal Endurance of FilmInsulated Round Magnet Wire," ASTM-D2307 -. 2013.

[20] M.R. Khowja, C. Gerada, G. Vakil, P. Wheeler and C. Patel, "Novel integrative options for passive filter inductor in high speed AC drives," in IECON 2016-42nd Annual Conference of the IEEE Industrial Electronics Society, pp. 1137-1142, 2016.

[21] M. Raza Khowja, C. Gerada, G. Vakil, P. Wheeler and C. Patel, " Integrated output filter inductor for permanent magnet motor drives. In IECON 2016-42nd Annual Conference of the IEEE Industrial Electronics Society (pp. 2827-2832). IEEE

[22] M.R. Khowja, C. Gerada, G. Vakil, R. Abebe, S. Odhano, C. Patel and P. Wheeler, "Novel Motor-Shaped Rotational Inductor for Motor Drive Applications," IEEE Trans.Ind.Electron., vol. 67, pp. 1844-1854, 2019.

[23] M.R. Khowja, G. Vakil, C. Gerada, T. Yang, S. Bozhko and P. Wheeler, "Trade-off Study of a High Power Density Starter-Generator for Turboprop Aircraft System," in IECON 2019-45th Annual Conference of the IEEE Industrial Electronics Society, pp. 1435-1440, 2019. 